\title{
Comparison among measures of depression: reliability, validity, relationship to anxiety and personality and the role of age and life events
}

\author{
Konstantinos Fountoulakis*1, Per Bech², Panagiotis Panagiotidis', \\ Sotiris Kantartzis ${ }^{1}$, Melina Siamouli ${ }^{1}$, Anna Papadopoulou ${ }^{1}$, \\ Marina Papadopoulou ${ }^{1}$, Apostolos Iacovides ${ }^{1}$ and George Kaprinis ${ }^{1}$
}

\author{
Address: ${ }^{13 r d}$ Department of Psychiatry, Aristotle University of Thessaloniki, Greece and ${ }^{2}$ Frederiksborg General Hospital Department of \\ Psychiatry, Hillerod Denmark \\ * Corresponding author
}

\author{
from International Society on Brain and Behaviour: 2nd International Congress on Brain and Behaviour \\ Thessaloniki, Greece. 17-20 November 2005 \\ Published: 28 February 2006 \\ Annals of General Psychiatry 2006, 5(Suppl I):S326 doi:I0.1 I86/I744-859X-5-SI-S326
}

\section{Background}

During the last decades, several scales assessing depressive symptoms emerged, however there are only a few studies comparing them in terms of reliability and validity.

\section{Materials and methods}

The study sample included 40 depressed patients $29.65 \pm$ 9.38 years old, and 120 normal comparison subjects $27.23 \pm 10.62$ years old. Clinical Diagnosis was reached by consensus of two examiners with the use of the SCAN v.2.0. The depressive scales applied and standardized were the CES-D, ZDRS, BDI-I, and the KSQ. Also, the STAI, the Life Events scale (Holms and Rahe), and the EPQ were administered. The analysis included the comparison of psychometric properties and the use of Pearson correlation coefficient and factor analysis.

\section{Results}

The results suggest that all scales correlated with anxiety measurements, sociodemographic variables, personality dimensions and non-significant indices to a similar extend. However, the MDI performed somewhat better, while the ZDRS had a very low internal consistency.

\section{Discussion}

The comparison of several depressive scales provided no impressive results on the superiority or inferiority of a specific scale on the others. 